\title{
Retrospective on the resource for radiative cooling
}

\author{
Paul Berdahl $\odot *$ \\ Lawrence Berkeley National Laboratory, Heat Island Group, Berkeley, California, \\ United States
}

\begin{abstract}
The history of quantitative measurements of radiative cooling is briefly reviewed, starting with Count Rumford in 1804. The cooling results from upward emission of thermal infrared radiation (wavelengths of 5 to $50 \mu \mathrm{m}$ ) that is not fully offset by downward atmospheric emission. The downward emission is characterized by the apparent atmospheric (sky) emittance and the surface air temperature. In 1984, an equation was published that describes the clear sky emittance as a function of the surface dew point temperature. At the time, this equation was merely one of many empirical relations. Now that time has passed, experimental and theoretical advances support its validity. Further refinements can include improved corrections for time-of-day and the lower air pressure at elevated locations. Complex computer codes for predicting atmospheric radiation have reached quantitative maturity. Given profiles of air temperature, water vapor, $\mathrm{CO}_{2}, \mathrm{O}_{3}, \mathrm{CH}_{4}, \mathrm{~N}_{2} \mathrm{O}$, and aerosols, they can compute spectral radiances with an accuracy of $\sim 3 \%$. The effect of clouds in reducing radiative cooling remains more uncertain. (c) 2021 Society of Photo-Optical Instrumentation Engineers (SPIE) [DOI: 10.1117/1.JPE $.11 .042106]$
\end{abstract}

Keywords: radiative cooling; radiative transfer; infrared spectroscopy; atmospheric radiation.

Paper 21066SS received Aug. 18, 2021; accepted for publication Sep. 22, 2021; published online Oct. 8, 2021.

\section{Introduction}

The internal temperature of the sun is 15 million $\mathrm{K}$ and decreases with distance from the center until, at the "surface," the temperature is roughly $6000 \mathrm{~K}$. At this temperature, electrons combine with nuclei to form neutral atoms and the sun's atmosphere becomes transparent. The resulting spectrum is that of a "black body" first described by Max Planck in 1900. If we have a passive macroscopic cavity that is in equilibrium at a uniform temperature $T$, then it contains a photon gas that has a Planck spectrum. The energy center of the $6000 \mathrm{~K}$ spectrum is about $700 \mathrm{~nm}$, at the boundary between the visible and infrared spectra. (A method to locate the energy center of the Planck spectrum is given in the Appendix.)

The temperature of the earth-slightly below $300 \mathrm{~K}$-is determined by the balance of incoming solar radiation absorbed by the earth system and outgoing thermal radiation. As this temperature is about $1 / 20^{\text {th }}$ of that of the sun, the energy center wavelength is about $20 \times 700 \mathrm{~nm}=14 \mu \mathrm{m}$. The atmosphere of the earth is largely opaque to thermal infrared radiation (the greenhouse effect), so in first approximation on the earth's surface we experience immersion in a near-300 $\mathrm{K}$ radiation environment. The availability of radiative cooling as a resource, of course, is related to the fact that the atmosphere partially transmits the thermal infrared, especially in the 8 to $13 \mu \mathrm{m}$ "atmospheric window."

The earth's outgoing thermal infrared radiation is emitted into space, which itself has a temperature near (just below) $3 \mathrm{~K}$. This temperature is about a factor of 100 below that of the earth, so that the energy center wavelength of its blackbody radiation is about $1.4 \mathrm{~mm}$, in the millimeter range. This is the famous microwave background energy believed to have been decoupled from matter when the universe was only 300,000 years old. The spectrally integrated form of Planck's law yields the familiar Stefan-Boltzmann law that the total energy flux is proportional to $T^{4}$. Thus, the background energy flux is about $10^{-8}$ times smaller than the $300 \mathrm{~K}$ flux, which can be neglected here.

*Address all correspondence to Paul Berdahl, paul.berdahl@gmail.com 
After the energy (oil) crisis of the early 1970s there was an upswelling in energy research. More efficient vehicles for transportation were sought, and solar energy applications were pursued. Photovoltaic solar cells, mostly of the silicon type, were investigated as a promising technology but (unlike today) the cost was roughly an order of magnitude too expensive for deployment. Buildings were understood as an important opportunity to conserve energy, as discussed at an American Physical Society Summer Study in 1974 and continually emphasized by Rosenfeld. ${ }^{1}$ Today, for example, we have efficient durable light emitting diodes to replace inefficient incandescent and fluorescent lamps. Vapor compression air conditioning, which makes life much more pleasant in hot regions, requires lots of electricity, and was therefore seen as a target for research. Various conferences ${ }^{2-6}$ examined radiative cooling, evaporative cooling, architectural approaches to convective cooling, etc. Physicists seemed particularly attracted to radiative cooling, influenced in part by an Italian group's pointing out ${ }^{7}$ that selective radiators using the atmospheric window can reach lower temperatures than non-selective gray-body radiators. This important paper also demonstrated a cooling effect during the day by shading the radiator from direct solar radiation. ${ }^{7}$ Since about $2014,{ }^{8}$ the availability of net cooling at noon even without a shade, due to solar reflectance above about 0.95 , has been a stimulus for renewed interest in the technology.

Characterization of atmospheric thermal radiation has led to the use of several different measures, in part because the spectrum is quite different from a blackbody spectrum. Fundamentally, we are concerned with the spectral radiance as a function of wavelength (or wavenumber) and as a function of zenith angle. The use of the total sky emittance as a key parameter is attractive since it is mainly independent of temperature. If the atmosphere was at a uniform temperature, its emittance would be precisely equal to 1 minus its transmittance, and transmittances are often nearly independent of temperature. As we shall see, the clear sky emittance can be reliably estimated based solely on the water vapor concentration. An alternative measure of the atmospheric radiation is the "sky temperature," which is the temperature a horizontal black or gray body will attain if it only exchanges radiant energy with the sky and is otherwise isolated from energy sources such as convection from ambient air. The absolute sky and air temperatures are related to the sky emittance $\varepsilon$ by $\varepsilon T_{\text {air }}^{4}=T_{\text {sky }}^{4}$. The resource for radiative cooling is directly related to the fact that $\varepsilon<1$ or, equivalently, that $T_{\text {sky }}<T_{\text {air }}$. The radiative cooling rate in $\mathrm{Wm}^{-2}$ is given by $\sigma(1-\varepsilon) T_{\text {air }}^{4}=\sigma\left(T_{\text {air }}^{4}-T_{\text {sky }}^{4}\right)$. Here $\sigma$ is the Stefan-Boltzmann constant. Sometimes the sky temperature depression, $\Delta T_{\text {sky }}=T_{\text {air }}-T_{\text {sky }}$, is used as a measure of the cooling resource. ${ }^{9}$ This parameter tends to be nearly independent of time of day as the air and sky temperatures rise and fall together. As an example, in the United States the summertime sky temperature depression along the humid southeastern gulf coast is only about $6^{\circ} \mathrm{C}$ to $8^{\circ} \mathrm{C}$, whereas in the dry desert southwest it is $18^{\circ} \mathrm{C}$ to $22^{\circ} \mathrm{C} .^{9}$

The sky is particularly cold in the 8 to $13 \mu \mathrm{m}$ window. Outside this window we measure mostly a blackbody spectrum at air temperature. A perfect spectral emitter with unity emittance in the window and zero emittance outside it cools at the same rate as a regular blackbody at air temperature but can theoretically reach much lower temperatures. The corresponding maximum $\Delta T$ for a horizontal radiator is about 2.4 times larger than $\Delta T_{\text {sky }} .{ }^{9}$ Of course, for radiator temperatures above air temperature, the selective radiator is inferior to a black body radiator.

The coldest part of the clear sky is the zenith since an instrument is looking through less atmosphere than near the horizon. Near $11 \mu \mathrm{m}$, the monthly mean clear zenith sky emittance is as low as about $0.1 .{ }^{10}$ Based on Planck's equation and using $300 \mathrm{~K}$ as a reference temperature, the radiant temperature is about $200 \mathrm{~K}$. Still lower radiant temperatures are to be expected at the earth's poles and on the top of mountains where telescopes are sited.

The sky temperature and sky emittance are useful parameters for making brief computations. But the sky temperature is only relevant for horizontally exposed black or gray bodies. And the sky emittance is only an "effective" parameter since the atmosphere is not at a uniform temperature. In complex situations, such as when the angular and/or spectral details of the radiation are important, one may need to revert to the full radiative transfer equations. Short of using a complete radiative code to simulate the sky radiance, if one knows the overall sky emittance, one can use empirical methods to estimate the spectral and angular components as, for example in Refs. 11 and 12. It is worth noting that in both these references, the spectral "transmittance" in the zenith direction is actually one minus the spectral emittance. 


\section{Historical Background for Radiative Cooling}

It has been known since antiquity that dew and frost form particularly readily in the open under clear skies. Also, minimum night time temperatures are lower under clear compared to cloudy skies.

Count Rumford published an early account on heat transfer by thermal radiation in $1804 .{ }^{13}$ He first studied the rate at which a cylinder containing hot water cooled and was surprised that a polished metal surface inhibited cooling relative to the same surface covered with a cloth. Thus, he was observing the low emittance of bare, clean metal surfaces. He also concluded that while heat convection by air was a factor, invisible heat radiation was quite important. Thus, hot surfaces could heat nearby objects by radiative transfer. He reasoned analogously that cold objects emitted frigorific radiation and found that a cold blackened metal surface could cool a nearby object more effectively than a bare cold metal surface. Of course, in the early 1900's Planck's law of thermal radiation and the invention of quantum mechanics made it clear that at absolute zero no heat radiation is emitted and that radiative cooling effects are due to the absence of heat radiation.

Here is the concluding paragraph of Count Rumford's paper:

The excessive cold which is known to prevail, in all seasons, on the tops of high mountains, and the frosts at night which frequently take place on the surface of the plains below, seem to indicate that frigorific rays arrive continually at the surface of the earth from every part of the heavens; and it is no doubt by the action of these rays that our planet is continually cooled, and enabled to preserve the same mean temperature for ages, notwithstanding the immense quantities of heat that are generated at its surface by the continual action of the solar rays. The action of these frigorific nocturnal rays will likewise justify the inhabitants of hot climates, who, in order to be more cool during their hours of rest, remove their beds in summer to the tops of their houses.

Thus, he has inferred that heat radiation leaving the earth balances solar heat gain and further that an effective passive technique for utilizing nocturnal radiative cooling is to sleep on a rooftop.

In $1828 \mathrm{~F}$. Arago wrote ${ }^{14}$ (translation below):

Si l'on place en plein air, dans une nuit calme et sereine, de petites masses d'herbe (grass), de coton, d'edredon (duck down), ou de toute autre substance filamenteuse, on trouve, apres un certain temps, que leur temperature est de 6 , de 7 et meme de 8 deg centigrade au-dessous de la temperature de atmosphere ambiante.

If one places outdoors, on a night that is calm and serene [and clear!], small quantities of grass, of cotton, of duck down, or of all other filamentary substances, one finds, after a certain time, that their temperature is $6 \mathrm{deg}, 7 \mathrm{deg}$, or even $8 \mathrm{deg}$ centigrade below that of the ambient air.

This observation that low-density fibrous materials can cool well below air temperature when exposed to the night sky is clearly a result of radiative cooling while the fibrous nature of the material helps insulate the cool fibers from convective warming by the ambient air.

Skipping forward by about 100 years to the quantitative work by Brunt in $1932,{ }^{15}$ based on measurements by Dines, ${ }^{16}$ it was found that the clear sky emittance can be written as

$$
\varepsilon_{0}=a+b p^{1 / 2}
$$

an equation still important today. As mentioned above, the sky emittance is a convenient shorthand for expressing the downward flux of thermal atmospheric radiation as $R_{\text {down }}=\varepsilon_{0} \sigma T_{\text {air }}^{4}$, where $\sigma$ is the Stefan-Boltzmann constant $\left(5.67 \times 10^{-8} \mathrm{~W} \mathrm{~m}^{-2} \mathrm{~K}^{-4}\right)$ and $T_{\text {air }}$ is the ambient air temperature in absolute degrees $(\mathrm{K})]$. Here $a$ and $b$ are dimensionless constants, and $p$ is the water vapor pressure in $\mathrm{mb}$. [1 $\mathrm{mb}=1$ hecto (hundreds of) pascal $=\mathrm{hPa}$ ].

Brunt's equation is purely empirical but can be made to fit better than alternative formulations. It fits data at specific zenith angles as well as the hemispherical average. However, the extrapolation to $p=0$ still represents an atmosphere with some (small) amount of water vapor that causes nearly complete opacity near $6 \mu \mathrm{m}$ and beyond $25 \mu \mathrm{m}$. Further, the extrapolation to large values of $p$ would suggest that the clear sky emittance can exceed unity-highly unlikely except in limited spectral ranges under temperature inversion conditions. The observed values of $p$ range from below $2.4 \mathrm{hPa}\left(\mathrm{dew}\right.$ point, $-12^{\circ} \mathrm{C}$ ) up to about $28 \mathrm{hPa}$ (dew point, $23^{\circ} \mathrm{C}$ ). 
In 1932, it was well known that water vapor was a major factor in the absorption of atmospheric radiation, as Brunt's equation demonstrates. It was also known that carbon dioxide caused strong absorption, particularly in the 14 to $16 \mu \mathrm{m}$ region, but the overlap between the $\mathrm{CO}_{2}$ and $\mathrm{H}_{2} \mathrm{O}$ bands impeded the recognition of carbon dioxide's future role in global warming.

Two additional key historical references are due to Australian A. K. Head and the French physicist F. Trombe. Head ${ }^{17}$ anticipated the theoretical work of the Italian group ${ }^{7}$ on the advantages of 8 to $13 \mu \mathrm{m}$ selective radiators, as documented in his patents. Trombe wrote an excellent review on radiative cooling, including the strategy of placing one radiative cooling panel inside another to reduce convective heat intrusion and reach lower temperatures. ${ }^{18}$

\section{More Recent History}

When we, at Lawrence Berkeley National Laboratory (Berkeley Lab) started work in the late 1970s to determine the resource for radiative cooling, we knew that the overall clear sky emittance could be expressed by a Brunt-type equation. For this reason, we initially focused on determining the angular and spectral distribution of the radiation. We soon learned that while the Brunt equation was in wide use, the value of the constants $a$ and $b$ varied widely. The textbook author Sellers ${ }^{19}$ was quite helpful in this regard. He looked at the results of 22 evaluations and took median results as $a=0.605$ and $b=0.048$. These specific values are now commonly cited. It is worth mentioning that while atmospheric thermal radiation varies from place to place due to climate variations, the laws of physics do not.

We made year-round measurements of atmospheric radiation at six sites in the warm southern half of the United States. The first four sites were Tucson, Arizona; St. Louis, Missouri; San Antonio, Texas; and Gaithersburg, Maryland. After collecting at least one summer's data at each of these locations, two sets of instruments were moved to Boulder City, Nevada and West Palm Beach, Florida, the purpose being to extend the range of humidity to very dry and very humid climates. Emphasis was on summer rather than winter data. Ultimately, 57 station months of useful data were collected.

The basic sky emittance measurements were obtained from the Eppley model PIR pygeometer. However, we used our specialized spectral and angular radiometer to adjust the pyrgeometers' calibrations by up to 0.03 in sky emittance. Our specialized instrument system was based on a Barnes Engineering 12-880 radiometer fitted with optical filters to isolate the 8 to $13 \mu \mathrm{m}$ atmospheric window, the $9.6 \mu \mathrm{m}$ ozone band, the minor window at 17 to $22 \mu \mathrm{m}$, etc. The basic radiometer was temperature controlled at $46^{\circ} \mathrm{C}$, had a pyroelectric detector, a coated germanium lens, and a gold-surfaced chopper. In a no-filter channel, the 8 to 13 window region was included as well as a broader spectral range which, however, was not spectrally uniform. The angularweighted sum of the no-filter channel was termed the "pseudo pyrgeometer." This pseudo pyrgeometer was used to guarantee that for complete low-level clouds-a limiting condition of thermal equilibrium - the Eppley instrument would indicate $\varepsilon_{0}=1$ when the pseudo pyrgeometer indicated the same. In turn, the specialized radiometer was calibrated against a variable temperature black body. At half hour intervals, the radiometer would view the sky at zenith angles of $0 \mathrm{deg}, 20 \mathrm{deg}, 40 \mathrm{deg}, 60 \mathrm{deg}$, and $80 \mathrm{deg}$ with northern azimuth. After viewing the sky, the instrument would then view the black body, which was mainly controlled at $70^{\circ} \mathrm{C}$, but allowed to gradually cool to near ambient temperature early in the morning. Least squares fitting yielded calibration constants for each channel and an estimate of the (small) emittance of the rotatable gold-coated viewing mirror. The presence and absence of clouds was determined from frequency distributions of spectral radiance, as the clear sky is notably colder than the cloudy sky. Further details are available in Ref. 20.

In support of Berkeley Lab's thermal infrared sky measurements, some numerical computations were performed with the Air Force Geophysics program's LOWTRAN 3B. ${ }^{21}$ The program used information about atmospheric temperature and composition to compute low-resolution $\left(20 \mathrm{~cm}^{-1}\right)$ spectral transmittances on arbitrary atmospheric paths. Due to spectral averaging, Beer's law of simple exponential decay does not hold (each spectral range includes differing spectral decay rates). However, local thermodynamic equilibrium holds and Kirchhoff's law does apply. Hence the thermal emission from a thin atmospheric layer can be inferred from one minus 
its transmittance times the Planck function. ${ }^{20}$ Reference 20 shows examples of computed spectrally and angularly resolved sky radiances. As time has passed, LOWTRAN has first been supplemented to calculate radiances as well as transmittance, and then been supplanted by moderate resolution transmittance ${ }^{22}$ and high resolution transmittance. ${ }^{23}$ The highest resolution modern codes are termed line-by-line, meaning they account for each of the numerous individual spectral absorption lines, a task made complex by the fact that the distant wings of the lines contribute significantly, requiring tabulation as continuum absorption spectra for $\mathrm{H}_{2} \mathrm{O}$ and $\mathrm{CO}_{2}$. A recent paper by $\mathrm{Li}$ and $\mathrm{Coimbra}^{24}$ provides some simplification of the mathematical problem while still maintaining high spectral resolution. They tabulate absorption spectra vs. wavenumbers (inverse of wavelength) from 0 to $2500 \mathrm{~cm}^{-1}$ ( $\infty$ to $4 \mu \mathrm{m}$ ) with resolution of $0.01 \mathrm{~cm}^{-1}$. That is, there are $2.5 \times 10^{5}$ data points for each species. See Fig. 4 in Ref. 24 and Figs. B1 and B2 in Ref. 25 to appreciate the complexity in the relevant spectra. As a benchmark reference for the current paper, Ellingson et al. ${ }^{26}$ reported median results from more than 30 radiation codes for the downwelling thermal infrared energy flux for five reference atmospheres.

The state of the art for radiation codes to simulate atmospheric radiative transfer has reached a maturity such that deviations from measurements are now often attributed to uncertainties in input data (e.g., for water vapor). Still, the codes are quite complex and subject to continuing refinement.

\section{Results of the Berkeley Lab Measurement Program}

The most important result from our program in the early 1980s is the clear sky emittance as a function of dew point temperature, ${ }^{9,27}$

$$
\varepsilon_{0}=0.711+0.56\left(T_{\mathrm{dp}} / 100\right)+0.73\left(T_{\mathrm{dp}} / 100\right)^{2},
$$

which is plotted in Fig. 1. Here $T_{\mathrm{dp}}$ is the dew point temperature in ${ }^{\circ} \mathrm{C}$. The use of $T_{\mathrm{dp}}$ rather than water vapor pressure $p$ is purely one of convenience; there is a one-to-one correspondence of these two variables. Two small adjustments were recommended. ${ }^{9}$ First, to account for the day/ night variation of the temperature profile, $\Delta \varepsilon_{t}=0.013 \cos [2 \pi(t / 24)]$ with $t$ the solar time; the emittance is slightly lower during the day. Second, in Ref. 9, based on work by Ref. 28, we introduced an additional small correction $\Delta \varepsilon_{p}=0.00012\left(p_{\text {atm }}-1000\right)$ to account for the reduction of atmospheric pressure $p_{\text {atm }}$ in mb at high-elevation sites. (Less air pressure causes lower molecular collision rates, which in turn narrows absorption lines.)

The other three curves in Fig. 1 are based on the Brunt form with $a=0.605, b=0.048$ of Sellers, ${ }^{19} a=0.612, b=0.044$ of Alados et al., ${ }^{29}$ and $a=0.585, b=0.057$ based on Li et al. ${ }^{30}$ from measurements of 7 US stations of NOAA's Surface Radiation Budget Network. In the Li et al. work, an unusual adjustment was made to the data that was reversed for presentation here.

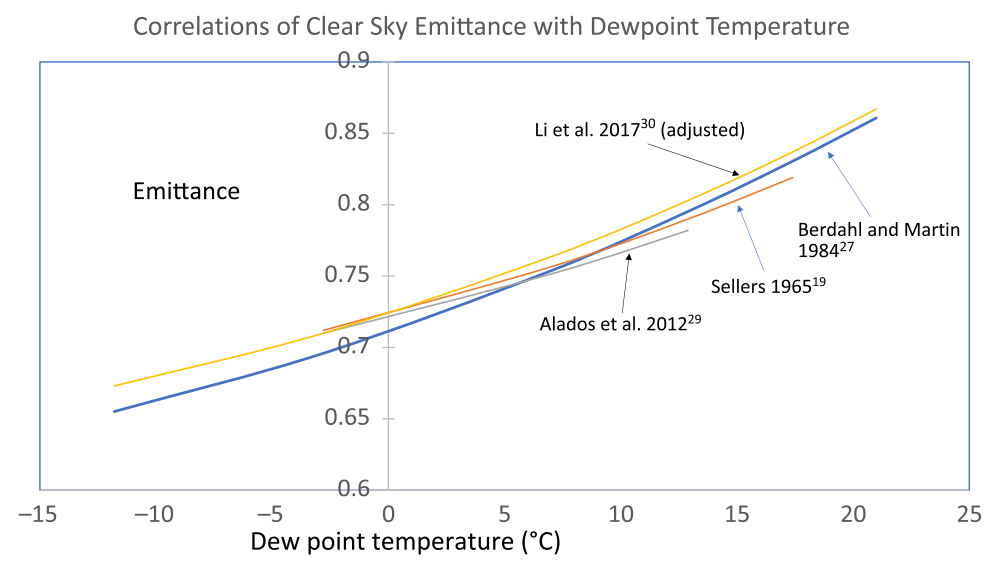

Fig. 1 Four curves, each of which represents clear sky emittance as a function of dew point temperature. 
Specifically, to approximately offset their adjustment using their factor $F \neq 1$ instead of $F=1$, the result for $\varepsilon_{0}$ was adjusted upward by $3.5 \%$ at $T_{\mathrm{dp}}=20^{\circ} \mathrm{C}$ and by $4.7 \%$ at $-10^{\circ} \mathrm{C}$, to yield $a=0.585, b=0.057$. For completeness, we note that Eq. (2), fit to the Brunt form, yields ${ }^{27}$ $a=0.564, b=0.059$. As can be seen in Fig. 1 , in the central range of $[0,15]^{\circ} \mathrm{C}$ in dew point temperature, all four curves agree closely with one another, spanning a range in $\varepsilon_{0}$ of 0.02 . Similar plots in $1980^{31}$ and $1982,{ }^{20}$ showing curves from both measurements and theoretical work, span a range of 0.08 or more.

The paper by Ellingson et al. ${ }^{26}$ is a very useful reference for computed clear sky emittance. While the latest theoretical work includes refinements such as updated spectra for the continuum water and $\mathrm{CO}_{2}$ absorption, the Ellingson paper is crystal clear as to the input data used. For example, the exact profiles of temperature and water vapor for the five model atmospheres are listed explicitly. Computer output from $30+$ computer codes was examined, and the median results reported. To further simplify comparisons, the aerosol absorption was set to zero. Li et al. ${ }^{25}$ discuss contributions from aerosols. They find that for a modest aerosol optical depth of 0.1 at $497.5 \mathrm{~nm}$, there is a surface contribution of about $2 \mathrm{Wm}^{-2}$ for humid conditions and $6 \mathrm{Wm}^{-2}$ for very dry conditions. Also of interest is that the computer results were obtained for a base case of $300 \mathrm{ppm}$ of $\mathrm{CO}_{2}$ (to be used here) and for a doubling to $600 \mathrm{ppm}$. Ellingson et al. found increases in the downward surface flux of $2.5 \mathrm{Wm}^{-2}$ for the dry subarctic winter atmosphere and $1.2 \mathrm{Wm}^{-2}$ for the humid tropical atmosphere. For the upward fluxes at the top of the atmosphere they found reductions of 1.6 and $3.1 \mathrm{Wm}^{-2}$ for the dry and humid cases. These last numbers are relevant to global warming. However, they do not include the atmospheric response, for example, the additional evaporation of $\mathrm{H}_{2} \mathrm{O}$.

With these various details established, we can now examine the comparison of computations with experiment as shown in Fig. 2. It shows excellent agreement with the experimental data, except for potential extrapolations for the very dry subarctic winter. This model atmosphere is the only one with a temperature inversion near the surface, and the experimental data we have extends only down to about $-12^{\circ} \mathrm{C}$ in dew (frost) point. Also of interest is that there is a slight trend in that the theoretical data points rise slightly compared to the empirical curve as the dew point temperature decreases.

The consequences of our Eq. (2) are further detailed in Figs. 3 and 4. Figure 3 shows the cooling power in $\mathrm{Wm}^{-2}$ experienced by a horizontally exposed ideal black body maintained at air temperature as a function of air and dew point temperatures, for clear skies. For a realistic system, one needs to reduce the cooling to account for surface emittance below 1 , the effect of a windscreen if used, solar absorption, etc. Also, it is worth pointing out that access to high cooling rates requires very dry air. See $\mathrm{Li}$ and Coimbra ${ }^{24}$ for comparable results, in their Figs. 8 and 9. Figure 4 shows the corresponding sky temperature depressions, $\Delta T_{\text {sky }}$. These show the

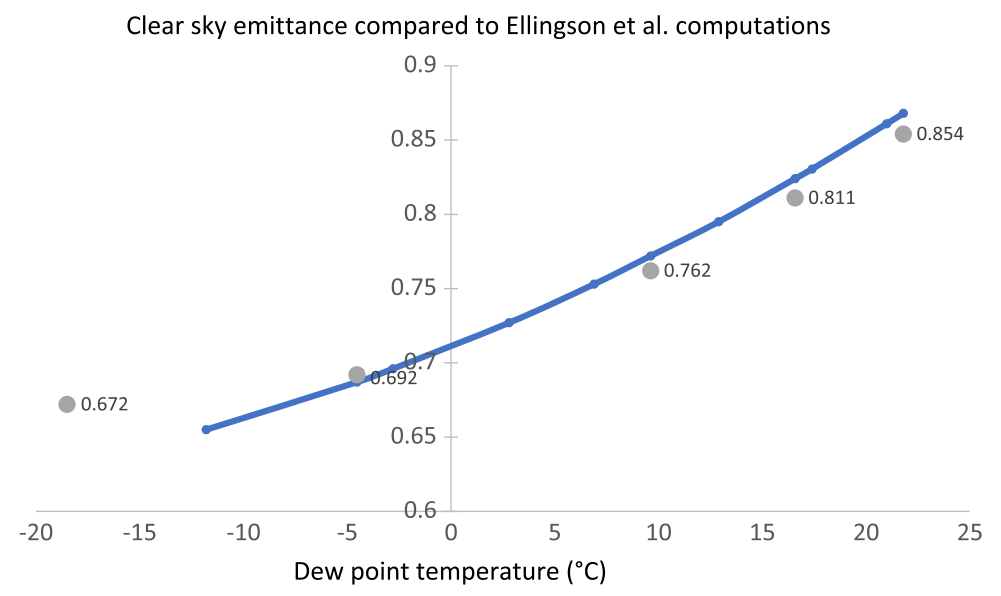

Fig. 2 Computed data for five model atmospheres compared with the empirical Eq. (2). The five model atmospheres and their surface temperatures in $\mathrm{K}$, from left to right are subarctic winter, 257.1; mid-latitude winter, 272.2; subarctic summer, 287.0; mid-latitude summer, 294.0; and tropical, 300.0 . 


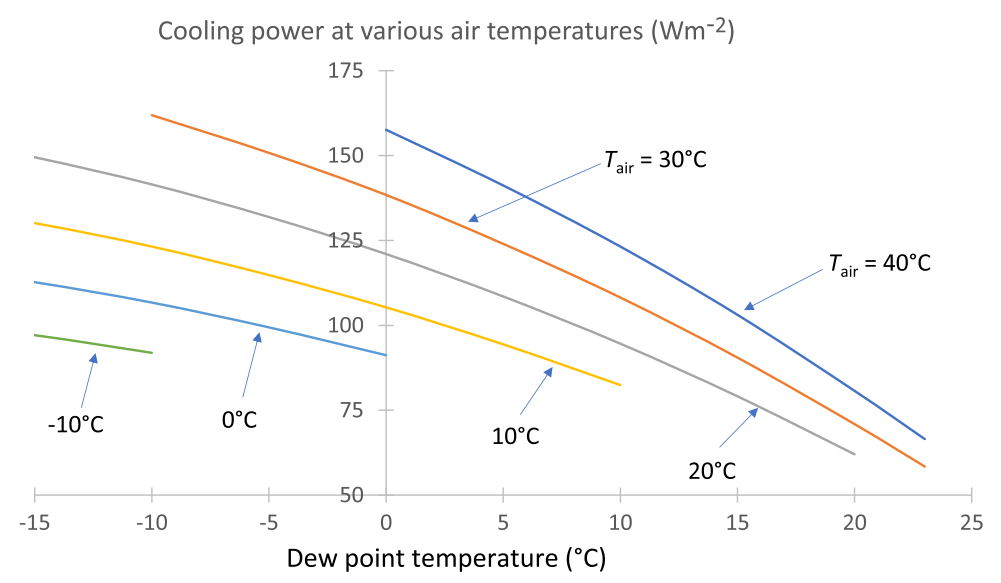

Fig. 3 Radiative cooling power under clear skies versus dew point temperature, at various surface air temperatures.

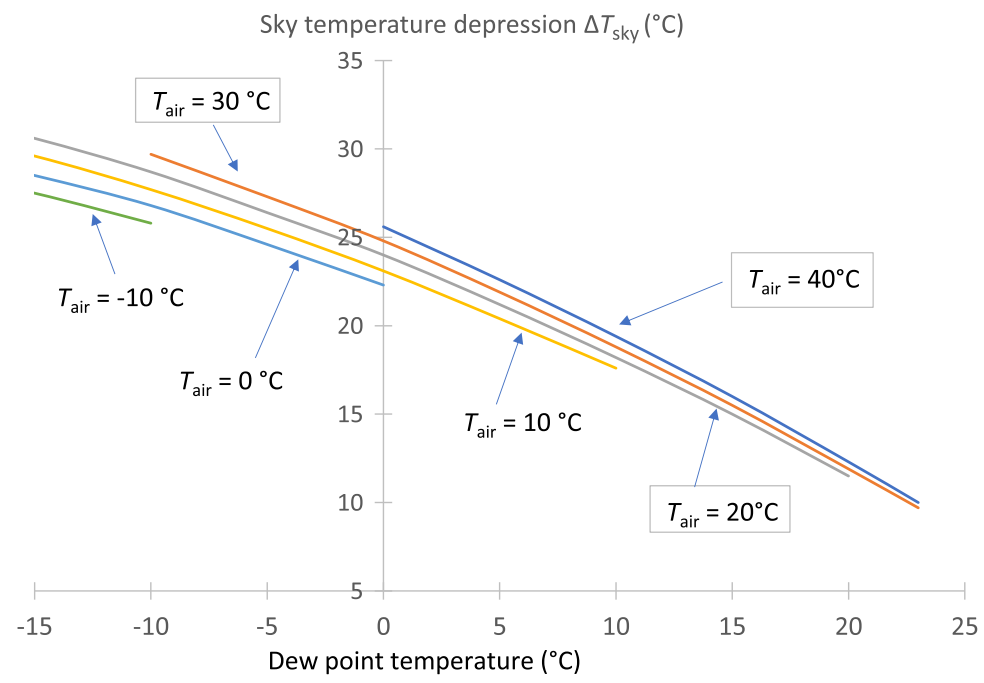

Fig. 4 Sky temperature depression under clear skies versus dew point temperature for various surface air temperatures.

maximum decrease in blackbody radiator temperature, below air temperature. For an ideal 8 to $13 \mu \mathrm{m}$ selective radiator, the corresponding temperature differences are larger by a factor of about 2.4. ${ }^{9}$

\section{Allowances for Cloud Cover}

Clouds always reduce the potential for radiative cooling below that for clear skies. Opaque clouds can be modeled as black bodies at the cloud base temperature. For very low clouds and fog, the observer is immersed in equilibrium heat radiation, $\Delta T_{\text {sky }}=0$. For low clouds, the sky temperature depression is roughly $1 / 3$ of the temperature difference between the cloud base temperature and the surface air temperature. ${ }^{32}$ The factor $1 / 3$ is related to the fact that only this fraction of the cloud's emission makes it down to the surface. More generally, we estimated using LOWTRAN $3 \mathrm{~B}^{9}$ that, for a complete opaque cloud layer, the sky emittance is

$$
\varepsilon=\varepsilon_{0}+\left(1-\varepsilon_{0}\right) \exp \left[-\left(T_{\text {air }}-T_{\text {cloudbase }}\right) / 46 \mathrm{~K}\right]
$$

High thin cirrus clouds are translucent and quite cold and can often be neglected in first approximation. The effects of typical broken cloud layers are complex and not easy to simulate. 


\section{Concluding Remarks}

The knowledge of the down-coming thermal radiation from the clear atmosphere is essential to estimate the performance of radiative cooling systems. Direct measurements with pyrgeometers are important but can be difficult due to issues with calibrations and the effects of sunlight upon the instruments. It is not an exaggeration to state that if the air is clear, estimates of atmospheric radiation using the air temperature and dew point can be preferred even though, of course, the estimates are themselves derived from pyrgeometer measurements.

Other applications of "sky temperatures" include input data for climatologists and meteorologists. Of course, the climatologist wants to know what the thermal infrared flux is throughout the atmosphere, not just at ground level, and wants the highest possible accuracy and spectral detail. The meteorologist may want to use the data to estimate night-time minimum temperatures. Another common application of sky temperatures is in the simulation of the energy performance of buildings. Outside roof and wall temperatures depend not just on solar radiation and air temperature but on the thermal infrared flux as well.

The order of magnitude of the cooling resource is $\sim 100 \mathrm{Wm}^{-2}$ under favorable circumstances, and it is available $24 \mathrm{~h}$ per day. On the other hand, the order of magnitude of the solar radiation resource is larger, at $1 \mathrm{kWm}^{-2}$ in full sun, which is itself often regarded as a dilute source. Thus, simple passive applications - such as the person with a bed (or solar-reflective tent) on the roof-have economic advantages. In recent years, the wide use of white coatings in hot climates to reduce unwanted solar heat gain while maintaining a high thermal emittance has been effective and economical. As Art Rosenfeld used to point out to us, if a white material costs the same as a more absorptive material, it is inherently cost effective. Recent years have also led to roofing materials companies developing higher-solar-reflectance materials that are not white. The companies have observed that some customers will not purchase white and pastel colors. Thus, they often employ darker pigments that at least have high near-infrared reflectance. Also, recently, fluorescent pigments have been considered to further reduce solar heat loads. Finally, traditional white materials and coatings have a visible reflectance of about $95 \%$ but only have a solar reflectance about $80 \%$ when the ultraviolet and near-infrared performance is considered. Thus, there is an important market opportunity for research and development of superior white materials.

This paper has been a historical look at the resource for radiative cooling. No attempt has been made here to review the latest literature on radiative cooling materials and systems. Two recent reviews on these subjects are given in Refs. 33 and 34.

Future work may extend the correlation of Eq. (2) to lower dew point temperatures and perhaps improve its accuracy. In the light of improvements in measurements and radiation codes over nearly four decades, it would be useful to revisit the time-of-day and atmospheric pressure corrections we derived in 1984. The pressure correction for elevated sites is based on old theoretical work ${ }^{28}$ and could likely be improved with new numerical calculations. The periodic timeof-day correction introduced by us ${ }^{9,20}$ has been appropriately criticized by Alados et al., ${ }^{29}$ who find that this correction should be larger in magnitude and that a time phase shift should be included. The time phase shift is related to the fact that peak air temperatures do not occur at solar noon but later in the day. For the magnitude of the time-of-day shift, we relied primarily on our pseudo-pyrgeometer, which is more sensitive to "window" radiation than non-window radiation. But the non-window regions of the spectrum are particularly sensitive to the lapse rate near the ground, so the Alados results are plausible.

\section{Appendix-The Energy Center of the Planck Blackbody Distribution}

The Planck distribution is rather broad. If we take the short- and long-wave ends as the 1 and 99 percent limits of energy the wavelength ratio is about a factor of 16 . For the $6000 \mathrm{~K}$ spectrum as a rough approximation for sunlight, we have the short-wave limit as $240 \mathrm{~nm}$ and the long-wave limit as $3.6 \mu \mathrm{m}$.

According to Bose-Einstein statistics, the occupation number for photons in each quantum state is simply $[\exp (x)-1]^{-1}$, where $x$ is the ratio of photon energy $E$ to the thermal energy $k_{\mathrm{B}} T$. The Planck distribution can then be derived by summing over the allowable photon momentum 
states, accounting for two photon polarizations and including a factor of $E$ to convert from occupation number to energy. If we now convert the sum over momentum to an integral over wavelength $\lambda$ we have for the total energy density $\sim \int d \lambda \lambda^{-5}[\exp (x)-1]^{-1}$ where the fact that $E=h c / \lambda$ has been used ( $h$ is Planck's constant, $c$ is the speed of light, $k_{\mathrm{B}}$ is Boltzmann's constant).

Now, an appropriate and traditional method for locating the "characteristic" wavelength of the distribution is to differentiate the integrand with respect to $\lambda$ and set to result to zero, thus locating the distribution's peak. One finds $1-\exp (-x)=x / 5$, the solution of which is $x=4.965$. Then $E=2.567 \mathrm{eV}, \lambda=483 \mathrm{~nm}$ for $6000 \mathrm{~K}$ radiation. On the other hand, the photon wavenumber $\left(\lambda^{-1}\right)$, proportional to the photon energy $E$, is also often used, particularly by spectroscopists, on the x-axis of plots of radiant energy distributions. Then we have an integral $\sim \int d E E^{3}[\exp (x)-1]^{-1}$. Differentiating this integrand with respect to $E$ and equating the result to zero, we find the location of the $E$-distribution's peak as given by $1-\exp (-x)=x / 3$. Here the solution is $x=2.821, E=1.459 \mathrm{eV}, \lambda=850 \mathrm{~nm}$, rather different from the peak in the $\lambda$-distribution.

The energy center of the Planck distribution offers yet another characteristic photon energy and wavelength for the distribution, which the present author prefers. It is simply the location for which half of the radiant energy is at shorter wavelength and half at longer wavelength. It does not depend on the choice of the abscissa. Numerically, ${ }^{20,35}$ we find that $x=3.500$, with $E=1.810 \mathrm{eV}, \lambda=685 \mathrm{~nm}$ for the $6000 \mathrm{~K}$ spectrum. For the $300 \mathrm{~K}$ spectrum, $\lambda=13.7 \mu \mathrm{m}$, for the $3 \mathrm{~K}$ spectrum, $\lambda=1.37 \mathrm{~mm}$.

\section{Acknowledgments}

I thank my co-authors Marlo Martin, Richard Fromberg, Eugene Clark, and Fateh Sakkal. Marlo was my primary collaborator, and he also provided liaison with the U. S. Dept. of Energy. Here at Berkeley Lab electronics technician Gerald Stoker assembled the equipment and kept it working. Brian Smith programmed the microcomputer that controlled the system, Fred Lenherr wrote the complex calibration software, and Peter Fuller interrogated the remote systems by telephone and provided post-processing. Since my retirement, my affiliation with LBNL has been hosted by R. Levinson. The work in the1980s was supported by the Assistant Secretary for Conservation and Renewable Energy of the U.S. Department of Energy under Contract No. DE-AC03-76 SF00098.

\section{References}

1. A. H. Rosenfeld, "An overview of energy conservation research at Lawrence Berkeley Laboratory," Energy Build. 1, 3-6 (1977).

2. In Proc. 2nd Nat. Passive Solar Conf., American Solar Energy Society, Philadelphia (1978).

3. In Proc. 3rd Nat. Passive Solar Conf., American Solar Energy Society, San Jose (1978).

4. In Proc. 4th Nat. Passive Solar Conf., American Solar Energy Society, Kansas City (1979).

5. In Proc. 5th Nat. Passive Solar Conf., American Solar Energy Society, Amherst (1980).

6. In Proc. Int. Passive and Hybrid Cooling Conf., American Section of the International Solar Energy Society, Miami Beach (1981).

7. S. Catalanotti et al., "The radiative cooling of selective surfaces," Solar Energy 17, 83-89 (1975).

8. A. P. Raman et al., "Passive radiative cooling below ambient air temperature under direct sunlight," Nature 515, 540-544 (2014).

9. M. Martin and P. Berdahl, "Characteristics of the infrared sky radiation in the United States," Solar Energy 33(3-4), 321-336 (1984).

10. M. Martin and P. Berdahl, "Summary of results from the spectral and angular sky radiation measurement program," Solar Energy 33(3-4), 241-252 (1984). 
11. P. Berdahl, M. Martin, and F. Sakkal, "Thermal performance of radiative cooling panels," Int. J. Heat Mass Transfer 26(6), 871-880 (1983).

12. C. G. Granqvist and A. Hjortsberg, "Radiative cooling to low temperatures: general considerations and application to selectively emitting SiO films," J. Appl. Phys. 52, 4205 (1981).

13. C. Rumford, "An enquiry concerning the nature of heat, and the mode of its communication," Philos. Trans. R. Soc. Lond., 1800-1814 1, 139-147 (1800-1814).

14. T. S. Eriksson and C. G. Granqvist, "Radiative cooling computed for model atmospheres," Appl. Opt. 21(23), 4381-4388 (1982). This reference contains our text's quotation from: F. Arago, Annuaire du Bureau des Longitudes pour l'an 1828, p. 149; reprinted in OEuvres Completes de Frangois Arago, Vol. 8 (Gide \& Weigel, Paris and Leipzig, 1858), p. 87.

15. D. Brunt, "Notes on radiation in the atmosphere," Quart. J. R. Meteor. Soc. 58, 389 (1932).

16. W. H. Dines and L. H. G. Dines, "Monthly mean values of radiation from various parts of the sky at Benson, Oxfordshire," Mem. R. Meteor. Soc. 2, 1 (1927).

17. A. K. Head, "Method and means for producing refrigeration by selective radiation," Australian patent 239364 (1959) and U.S. patent 3043112 (1962).

18. F. Trombe, "Perspectives sur l'utilization des rayonnments solaries et terrestres dans certains regions du monde," Rev. Gen. Therm. 6, 1285 (1967).

19. W. D Sellers, Physical Climatology, University of Chicago Press (1965).

20. P. Berdahl and R. Fromberg, "The thermal radiance of clear skies," Solar Energy 29(4), 299-314 (1982).

21. J. E. A. Selby, E. P. Shettle, and R. A. McClatchey, "Atmospheric transmittance from 0.25 to 28.5 um: supplement LOWTRAN 3B," U. S. Air Force Geophysical Laboratory, Hanscom AFB, Massachusetts, AFGL-TR-76-0258 (1976).

22. A. Berk et al., "MODTRAN6: a major upgrade of the MODTRAN radiative transfer code," Proc. SPIE 9088, 90880H (2014). See modtran.spectral.com for a commercial MODTRAN website provided by Spectral Sciences, Inc. An educational version can be found at climatemodels.uchicago.edu/modtran/.

23. I. E. Gordon et al., "The HITRAN6 molecular spectroscopic database," J. Quant. Spectrosc. Radiat. Transfer 203, 3-69 (2017). See hitran.org for HITRAN online.

24. M. Li and C. F. M. Coimbra, "On the effective spectral emissivity of clear skies and the radiative cooling potential of selectively designed materials," Int. J. Heat Mass Transfer 135, 1053-1062 (2019).

25. M. Li, Z. Liao, and C. F. M. Coimbra, "Spectral model for clear sky atmospheric longwave radiation," J. Quant. Spectrosc. Radiat. Transfer 209, 196-211 (2018).

26. R. G. Ellingson, J. Ellis, and S. Fels, "The intercomparison of radiation codes used in climate models: long wave results," J. Geophys. Res. 96(D5), 8929-8953 (1991).

27. P. Berdahl and M. Martin, "Emissivity of clear skies," Solar Energy 32(5), 663-664 (1984).

28. D. Staley and G. Jurica, "Effective atmospheric emissivity under clear skies," J. Appl. Meteor. 11, 349 (1972).

29. I. Alados, I. Foyo-Moreno, and L. Alsdos-Arboledas, "Estimation of downwelling longwave irradiance under all-sky conditions," Int J. Climatol. 32, 781-793 (2012).

30. M. Li, Y. Jiang, and C. F. M. Coimbra, "On the determination of atmospheric longwave irradiance under all-sky conditions," Solar Energy 144, 40-48 (2017).

31. E. Clark and P. Berdahl, "Passive cooling applications handbook," in Amherst Passive Solar Cooling Conf., LBL publication Pub-375, pp. 167-201, (1980).

32. P. Berdahl and M. Martin, "Thermal radiance of skies with low clouds," in Proc. Int. Passive and Hybrid Cooling Conf., Miami Beach, pp. 266-269 (1981).

33. D. Zhao et al., "Radiative sky cooling: fundamental principles, materials, and applications," Appl. Phys. Rev. 6, 021306 (2019).

34. A. S. Farooq et al., "Emerging radiative materials and prospective application of radiative sky cooling - a review," Renew. Sustain. Energy Rev. 144, 110910 (2021).

35. F. Kreith and M. S. Bohn, Principles of Heat Transfer, 4th ed., HarperCollins Publishers (1986). 
Paul Berdahl is a physicist (PhD, Stanford University). He joined Berkeley Lab in 1976 to work on solar resource assessment and assessment of the resource for radiative cooling. In the area of cool roofing, he has investigated the optical properties of pigments, soil, and heterogeneous surfaces. Recently, he studied fluorescent pigments to cool sunlit surfaces. His other research interests include the thermodynamics of infrared emission from semiconductors, pigments to reflect the infrared radiation from fire, and the high-temperature superconductors. 